\title{
Assessment of the validity of the 28-joint disease activity score using erythrocyte sedimentation rate (DAS28-ESR) as a disease activity index of rheumatoid arthritis in the efficacy evaluation of 24-week treatment with tocilizumab: subanalysis of the SATORI study
}

\author{
Norihiro Nishimoto $\cdot$ Nobuhiro Takagi
}

Received: 24 February 2010/Accepted: 31 May 2010/Published online: 10 July 2010

(C) Japan College of Rheumatology 2010

\begin{abstract}
As tocilizumab (TCZ) greatly inhibits inflammatory markers, methods of evaluating rheumatoid arthritis (RA) disease activity that include inflammatory markers may overestimate the effect of TCZ treatment. We have evaluated the impact of inflammatory markers on the efficacy of TCZ by comparing the efficacy indicated by the 28 joint disease activity score using erythrocyte sedimentation rate (DAS28-ESR) with that indicated by the clinical and simplified disease activity indexes (CDAI and SDAI, respectively) and the American College of Rheumatology (ACR) core set criteria in a double-blind study of TCZthe SATORI study. The Spearman correlation coefficient between DAS28-ESR and CDAI was comparable between that at week 24 and that at baseline [correlation coefficient at baseline and week 24 was $0.823(p<0.0001)$ and 0.818 $(p<0.0001)$, respectively]. A large difference between the DAS28 remission rate and CDAI remission rate was observed at week 24 . However, these results are comparable to those of a previous study conducted with nonTCZ-treated patients. Moreover, the same results were obtained in the comparison between the DAS28-ESR and SDAI, even though the SDAI includes an inflammatory parameter as a component. These results confirm that the DAS28-ESR has a validity comparable to that of other methods in terms of evaluating the RA treatment efficacy of TCZ, despite its strong inflammatory marker-inhibiting effects.
\end{abstract}

N. Nishimoto $(\bowtie)$

Laboratory of Immune Regulation, Wakayama Medical

University, 105 Saito Bio Innovation Center, 7-7-20 Saito-Asagi,

Ibaraki, Osaka 567-0085, Japan

e-mail: norichan@wakayama-med.ac.jp

N. Takagi

Chugai Pharmaceutical Co., Ltd., Tokyo 103-8324, Japan
Keywords Interleukin $6 \cdot$ Clinical disease activity index · Simplified disease activity index - Acute phase protein . Inflammatory markers

\section{Introduction}

Tocilizumab (TCZ) is a monoclonal anti-interleukin-6 (IL-6) receptor antibody that binds to cell membranebound IL-6 receptors and to free soluble IL-6 receptors in the serum, thereby blocking IL-6 signalling into cells [1]. This drug was developed to treat patients with rheumatoid arthritis (RA), Castleman's disease and juvenile idiopathic arthritis, and has been approved as a treatment for these indications in Japan [2-9]. It has been reported that treatment with TCZ alone or in combination with disease-modifying antirheumatic drugs (DMARDs), including methotrexate (MTX), has therapeutic effects in RA patients who had an inadequate response to anti-tumour necrosis factor (TNF) therapy or non-biologic DMARDs or in those who are MTX naive or had an inadequate response to MTX [2-13].

TCZ directly inhibits the production of acute phase proteins, such as C-reactive protein (CRP) and fibrinogen from hepatocytes, by directly inhibiting the action of IL-6. Consequently, the CRP level and erythrocyte sedimentation rate (ESR) rapidly and intensively decrease with the initiation of TCZ treatment before any improvement in swollen or tender joint counts (TJCs) is observed [14], possibly resulting in a discrepancy between an improvement in inflammatory markers and an improvement in actual RA disease activity. This has led to concern among clinicians on the possibility that methods of evaluating RA disease activity that include CRP and ESR may overstate the therapeutic effect of TCZ treatment for RA compared to methods that do not. 
To investigate the validity of the 28-joint Disease Activity Score using ESR (DAS28-ESR) for evaluating the efficacy of TCZ as a treatment for RA, we compared the efficacy assessment in the SATORI study (a double-blind, comparative study of TCZ conducted in Japan) [4] using the DAS28-ESR [15] with the efficacy assessments using the clinical disease activity index (CDAI) [16], simplified disease activity index (SDAI) [17] and American College of Rheumatology (ACR) response [18].

\section{Patients and methods}

\section{Patients}

This study was based on data from the SATORI study that has been previously published [4]. The inclusion and exclusion criteria for enrolment in the SATORI study were as follows. Eligible patients were between 20 and 75 years old, fulfilled the ACR (formerly, the American Rheumatism Association) 1987 revised criteria for the classification of RA and had disease duration $>6$ months. All candidates had been treated with MTX ( $8 \mathrm{mg} /$ week) for at least 8 weeks prior to enrolment but showed an inadequate response to MTX at enrolment by the persistence of active disease, defined as $\geq 6$ tender joints (of 49 evaluated), $\geq 6$ swollen joints (of 46 evaluated) and $\mathrm{ESR} \geq 30 \mathrm{~mm} / \mathrm{h}$ or $\mathrm{CRP} \geq 10 \mathrm{mg} / \mathrm{L}$. Patients were excluded if, immediately prior to the initiation of TCZ treatment (start of study), they had received anti-TNF agents or leflunomide within 12 weeks, plasma exchange therapy or surgical treatment within 4 weeks, or DMARDs other than MTX or immunosuppressants within 2 weeks. Patients who had received oral corticosteroids ( $\leq 10 \mathrm{mg} /$ day prednisolone) were enrolled if the dosage had not been changed within the 2 week period immediately preceding the start of $\mathrm{TCZ}$ treatment. Eligible patients had a white blood cell count $\geq 3.5 \times 10^{9} / \mathrm{L}$, a lymphocyte count $\geq 0.5 \times 10^{9} / \mathrm{L}$ or a platelet count of at least the lower limit of normal as defined by the local laboratory used for all analyses. Patients were excluded if they were functional class IV according to Steinbrocker's criteria [19], had aspartate transaminase, alanine transaminase or serum creatinine $\geq 1.5$ times the upper limit of normal, were positive for hepatitis B surface antigen and/or hepatitis $\mathrm{C}$ virus antibody, had pulmonary fibrosis or active pulmonary disease, had a history of serious adverse drug reaction to MTX, had concomitant pleural effusion, ascites or varicella infection or were excessive users of alcohol on a regular basis. Patients were excluded if they had a history of serious allergic reaction, or if they had significant cardiac, blood, respiratory system, neurologic, endocrine, renal, hepatic or gastrointestinal disease or an active infection requiring medication within 4 weeks before the start of treatment. Sexually active premenopausal women were required to have a negative urine pregnancy test result at the time of entry to the study and to use effective contraception throughout the study period.

\section{Protocol}

The following is a summary of the protocol of the SATORI study [4]. This trial is registered at http://www. clinicaltrials.gov (NCT00144521). The first patient was enrolled on 27 January 2004, and the last patient exited the study on 15 February 2005. Patients were randomly assigned to receive one of the following treatments for 24 weeks: (1) TCZ ( $8 \mathrm{mg} / \mathrm{kg})$ once every 4 weeks plus MTX placebo (TCZ group); (2) or TCZ placebo plus MTX $8 \mathrm{mg} /$ week (control group). Randomization was achieved by centralized allocation at a patient enrolment center. The dosage of TCZ used in this study was chosen based on the results of an earlier dose-finding study; the dose of MTX was the maximum dose allowed in Japan. Oral corticosteroids equivalent to $<10 \mathrm{mg} /$ day prednisolone were allowed, but the dose could not be increased during the study. Intra-articular injections of corticosteroid (only one joint at one treatment) and hyaluronate preparations were allowed. The use of one nonsteroidal anti-inflammatory drug (NSAID), including switching to another NSAID, was allowed. DMARDs, intravenous or intramuscular corticosteroids, plasmapheresis and surgical treatment were not allowed.

\section{Efficacy evaluation}

Efficacy was evaluated every 4 weeks by calculating the DAS28-ESR, CDAI and SDAI scores and the ACR response category. Changes in DAS28-ESR, CDAI and SDAI from baseline to week 24 were also calculated, and the DAS28ESR and ACR response categories were compared qualitatively [20]. Remission was evaluated using the following remission criteria, and the results were compared: DAS28$\mathrm{ESR}<2.6 ; \mathrm{SDAI} \leq 3.3 ; \mathrm{CDAI} \leq 2.8$ [21-23]. ACR20, ACR50 and ACR70 responses are defined as 20, 50 and 70\% improvement, respectively, in the tender joint count (TJC) and swollen joint count (SJC), and 20, 50 and $70 \%$ improvement, respectively, in at least three of the following five parameters: (1) patient self-assessed function (health assessment questionnaire); (2) patient global assessment (PGA); (3) physician global assessment (MDGA); (4) patient pain assessment; (5) either ESR or CRP.

The following formulae were used to calculate DAS28ESR, SDAI and CDAI, respectively: (1) DAS28-ESR = $0.56 \sqrt{ } \mathrm{TJC}+0.28 \sqrt{ } \mathrm{SJC}+0.70 \ln (\mathrm{ESR})+0.014 \mathrm{PGA}$ $(\mathrm{mm}) ;(2) \mathrm{SDAI}=\mathrm{SJC}+\mathrm{TJC}+\mathrm{PGA}(\mathrm{cm})+\mathrm{MDGA}$ $(\mathrm{cm})+\mathrm{CRP}(\mathrm{mg} / \mathrm{dL})$; (3) $\mathrm{CDAI}=\mathrm{SJC}+\mathrm{TJC}+\mathrm{PGA}$ $(\mathrm{cm})+$ MDGA $(\mathrm{cm})$, where SJC and TJC are the respective counts for 28 joints. 
Statistical analysis

Change in continuous variables (DAS28-ESR, CDAI and SDAI) is amenable to simple linear regression analysis; therefore, the latter two variables were compared by linear regression with DAS28-ESR as the independent variable. The probability of a significant association between the independent and dependent variables was defined as $p<0.05$.

\section{Results}

Time courses of disease activity scores and their components

Fifty-three of the 61 patients enrolled in the TCZ-treatment group in the SATORI study completed the study (24 weeks), and disease activity was measured in 53 patients at various time points. Figure 1 shows the time courses of the DAS28-ESR, CDAI and SDAI values and each of the evaluation items used to calculate these indices. DAS28ESR tended to progressively improve during the TCZ treatment, with the mean DAS28-ESR improving from 6.06 at baseline to 2.77 at week 24. The CDAI and SDAI also progressively improved during the TCZ treatment. Furthermore, each of the evaluation items, with the exception of ESR and CRP, used to calculate DAS28-ESR, CDAI and SDAI progressively improved during the TCZ treatment. Conversely, the ESR and CRP levels rapidly decreased from week 0 to week 4 , following which they remained approximately constant (Fig. 1). At week 24, joint swelling in the 28 joints of the DAS28 had completely improved (i.e., $\mathrm{SJC}=0$ ) in 13 patients $(24.5 \%)$, and joint tenderness had completely improved (i.e., TJC $=0$ ) in 20 patients $(37.7 \%)$. The sum of the SJC and TJC at week 24 was $\leq 1$ in 13 patients $(24.5 \%)$.
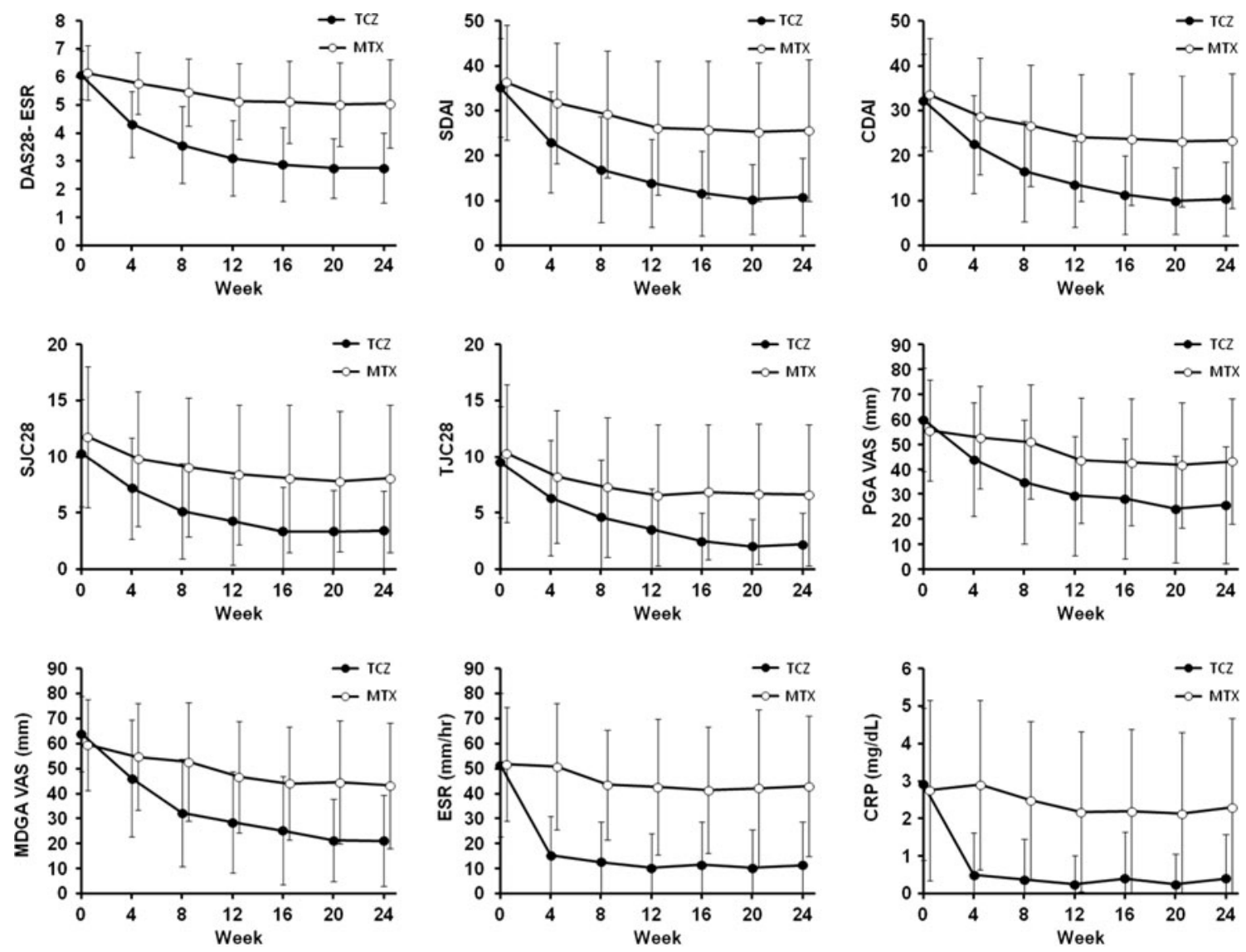

Fig. 1 Change in the indices of rheumatoid arthritis (RA) disease activity and in the variables used to calculate those indices over the 24 -week study. The indices and variables shown are the 28 -joint disease activity score using erythrocyte sedimentation rate (DAS28ESR), the clinical and simplified disease activity indices (CDAI and SDAI, respectively), 28-joint swollen and tender joint counts (SJC28 and TJC28, respectively), visual analog scale (VAS) of patient and physician global assessment (PGA and MDGA, respectively), C-reactive protein (CRP) and ESR. The treatment received was either tocilizumab (TCZ) or methotrexate (MTX). Mean values and standard deviations are shown. The data of the TCZ group and control group are shown as mean values among the 53 TCZ patients and 37 MTX patients who completed the 24-week study 
Correlations between DAS28-ESR and CDAI or SDAI, and between DAS28-CRP and CDAI or SDAI

Figure $2 \mathrm{a}$ is a scatter plot of the results for each patient in the TCZ group and shows the correlations between
DAS28-ESR and CDAI at baseline and at week 24, and between the change in DAS28-ESR and change in CDAI from baseline to week 24. The Spearman coefficient of correlation between DAS28-ESR and CDAI at week 24 was $0.823(p<0.0001)$, which was comparable to that at
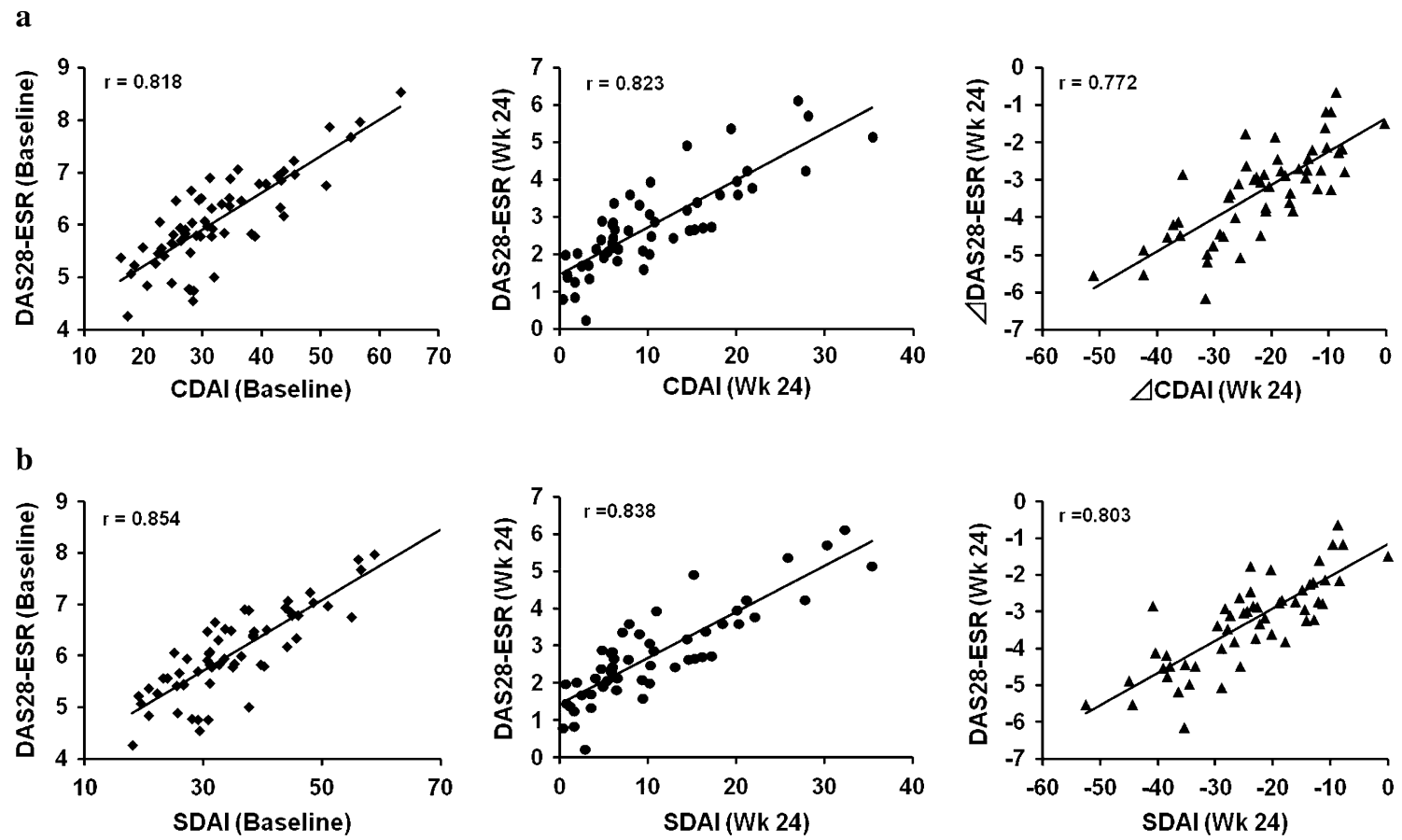

c
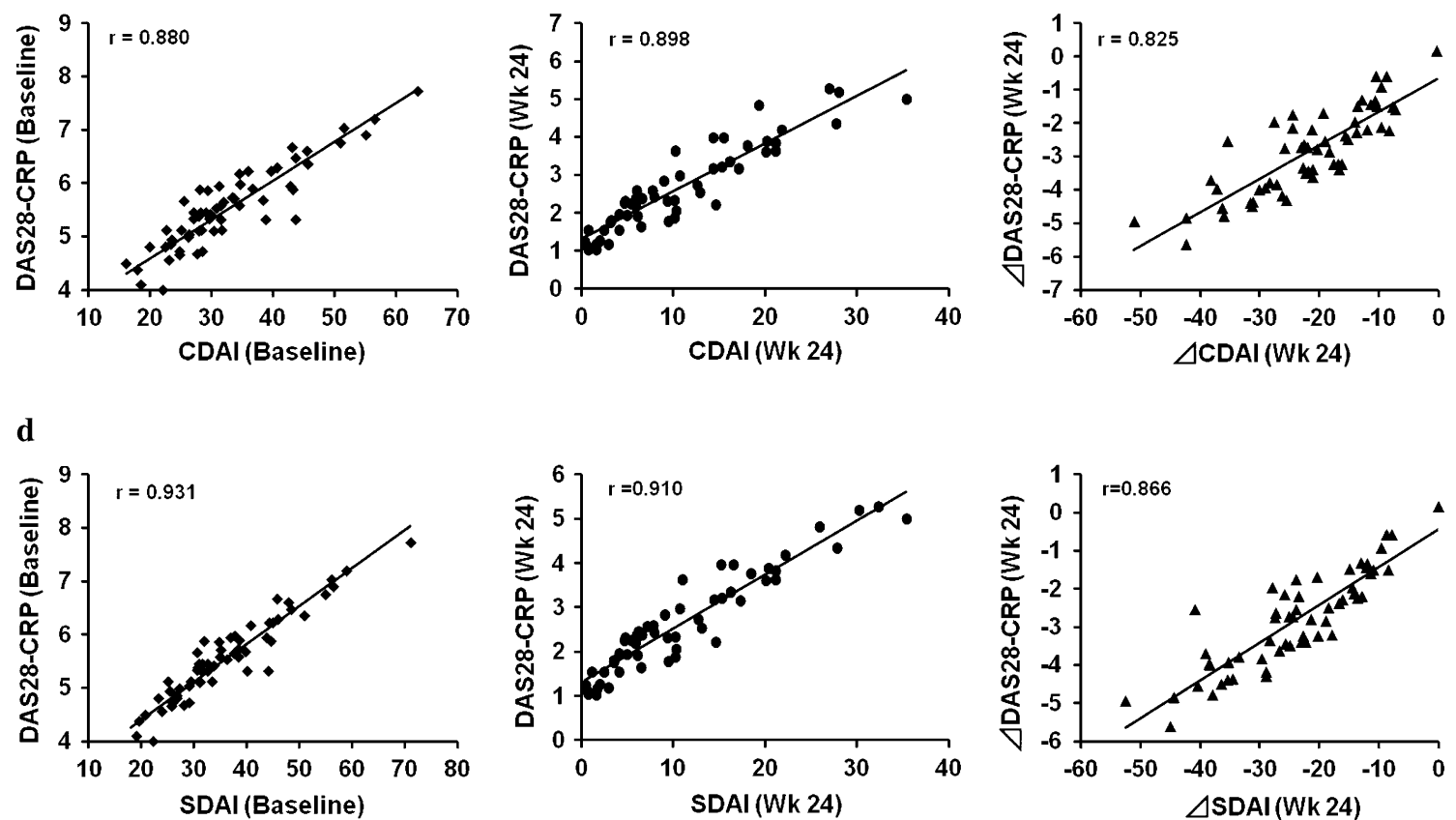

Fig. 2 Scatter plots of DAS28-ESR/DAS28-CRP versus CDAI/SDAI at baseline and at week 24, and change in DAS28-ESR/DAS28-CRP versus change in CDAI/SDAI at week 24 in the TCZ group. The results of the 61 patients at baseline and the 53 patients who completed the 24-week study in the TCZ group are shown. All regression lines indicate a highly significant correlation between DAS28-ESR/DAS28-CRP and CDAI/SDAI. a DAS28-ESR vs. CDAI, b DAS28-ESR vs. SDAI, c DAS28-CRP vs. CDAI, d DAS28-CRP vs. SDAI 
a
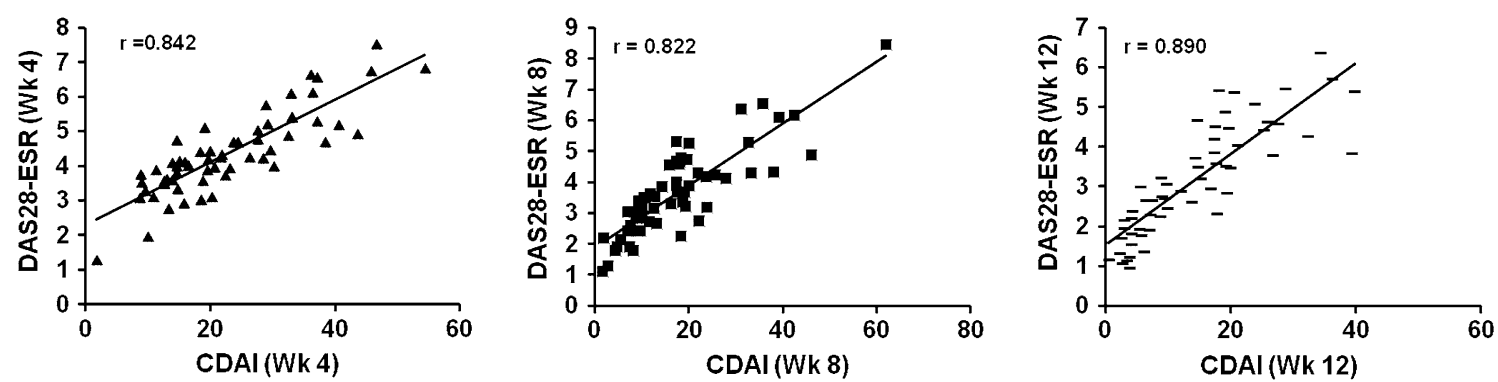

b
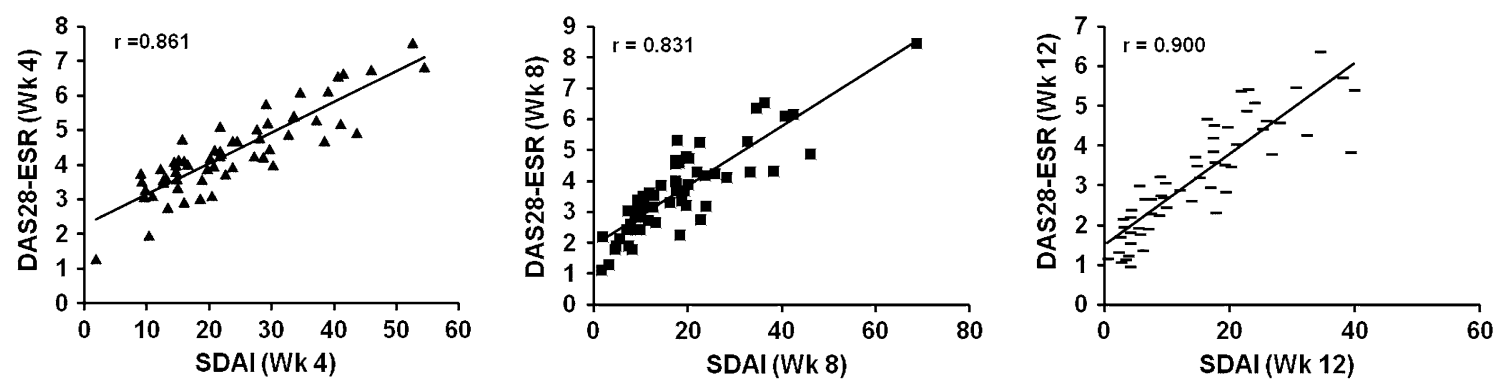

Fig. 3 Scatter plots of DAS28-ESR versus CDAI/SDAI at weeks 4, 8, and 12 in the TCZ group. The results of 58 patients at week 4, 60 patients at week 8 , and 59 patients at week 12 in the TCZ group are shown. The difference in patient numbers is due to missing ESR data. All regression

baseline $(0.818, p<0.0001)$. The coefficient of correlation between the change in DAS28-ESR and change in CDAI from baseline to week 24 was $0.772(p<0.0001)$.

The Spearman coefficient of correlation between DAS28-ESR and SDAI at week 24 was $0.838(p<0.0001)$, which was comparable to that at baseline $(0.854, p<0.0001)$. The coefficient of correlation between the change in DAS28ESR and the change in SDAI from baseline to week 24 was $0.803(p<0.0001)$ (Fig. 2b).

Because the DAS28-CRP is also frequently used to evaluate the disease activity of RA and because the CRP level is also considerably decreased by TCZ, a similar analysis was performed between DAS28-CRP and CDAI as well as between DAS28-CRP and SDAI. As shown in the Fig. $2 \mathrm{c}$ and d, the results were essentially the same as those between the DAS28-ESR and CDAS or SADI, although Spearman coefficient of correlation was always higher (Fig. 2c, d).

During the initial phase of TCZ treatment, rheumatologists frequently observe a rapid decrease in inflammatory markers, such as CRP and ESR, while the arthritis shows a more gradual improvement. To determine whether or not the DAS28-ESR and the other indices may become separated in the relatively early phase of the TCZ treatment, we also examined the correlation between DAS28-ESR and CDAI/ SDAI at weeks 4, 8, and 12 (Fig. 3; Table 1). The Spearman coefficient of correlation between DAS28-ESR and CDAI lines indicate a highly significant correlation between DAS28-ESR and CDAI/SDAI. a DAS28-ESR vs. CDAI (week 4, 8, 12), b DAS28-ESR vs. SDAI (week 4, 8, 12)

Table 1 Spearman coefficient of correlation between DAS28-ESR and CDAI or SDAI during treatment with TCZ or MTX

\begin{tabular}{lccccc}
\hline $\begin{array}{l}\text { Indices of disease } \\
\text { activity }\end{array}$ & \multicolumn{5}{l}{ Weeks after the first treatment } \\
\cline { 2 - 6 } & 0 & 4 & 8 & 12 & 24 \\
\hline \multicolumn{2}{l}{ DAS28-ESR vs. CDAI } & & & & \\
$\quad$ TCZ & 0.818 & 0.842 & 0.822 & 0.890 & 0.823 \\
MTX & 0.883 & 0.865 & 0.911 & 0.921 & 0.887 \\
DAS28-ESR vs. SDAI & & & & \\
TCZ & 0.854 & 0.861 & 0.831 & 0.900 & 0.838 \\
MTX & 0.893 & 0.869 & 0.902 & 0.930 & 0.897 \\
\hline
\end{tabular}

DAS28-ESR 28-joint disease activity score using erythrocyte sedimentation rate, CDAI, SDAI, clinical and simplified disease activity indices, respectively, $T C Z$ tocilizumab, MTX methotrexate

was almost constant during the treatment period, and there was no significant decrease in week 4 or 8 . The data were essentially the same as those between DAS28-ESR and SDAI in the TCZ treatment and those in the control MTX treatment (Table 1). The same results were also obtained from DAS28-CRP and CDAI and SDAI (data not shown).

Comparison between DAS28-ESR remission and CDAI or SDAI remission

Although DAS28-ESR well correlated with CDAI and SDAI, the rate of remission based on the DAS28-ESR 
Table 2 Remission and clinical variables in the tocilizumabtreated group of the SATORI study at week 24

SJC swollen joint count, TJC tender joint count

Fig. 4 Histograms of CDAI remission variables in the 17 patients who were in remission according to the DAS28 but not according to the CDAI

\begin{tabular}{|c|c|}
\hline Variable & $n(\%)$ \\
\hline DAS28-ESR $<2.6$ ( $\%$ of patients who participated in the full 24 -week study) & $25(47.2)$ \\
\hline SJC in 28 joints $=0$ in DAS remitters ( $\%$ in DAS remitters) & $11(44.0)$ \\
\hline TJC in 28 joints $=0$ in DAS remitters ( $\%$ in DAS remitters) & $17(68.0)$ \\
\hline SJC + TJC in 28 joints $\leq 1$ in DAS remitters (\% in DAS remitters) & $12(48.0)$ \\
\hline CDAI $<2.8$ (\% of patients who participated in the full 24 -week study) & $8(15.1)$ \\
\hline SJC in 28 joints $=0$ in CDAI remitters ( $\%$ in CDAI remitters) & $7(87.5)$ \\
\hline TJC in 28 joints $=0$ in CDAI remitters ( $\%$ in CDAI remitters) & $8(100)$ \\
\hline $\mathrm{SJC}+\mathrm{TJC}$ in 28 joints $\leq 1$ in CDAI remitters ( $\%$ in CDAI remitters) & $7(87.5)$ \\
\hline SDAI $<3.3$ ( $\%$ of patients who participated in the full 24 -week study) & $9(17.0)$ \\
\hline SJC in 28 joints $=0$ in SDAI remitters ( $\%$ in SDAI remitters) & $8(88.9)$ \\
\hline TJC in 28 joints $=0$ in SDAI remitters ( $\%$ in SDAI remitters) & $9(100)$ \\
\hline SJC + TJC in 28 joints $\leq 1$ in SDAI remitters ( $\%$ in SDAI remitters) & $8(88.9)$ \\
\hline
\end{tabular}
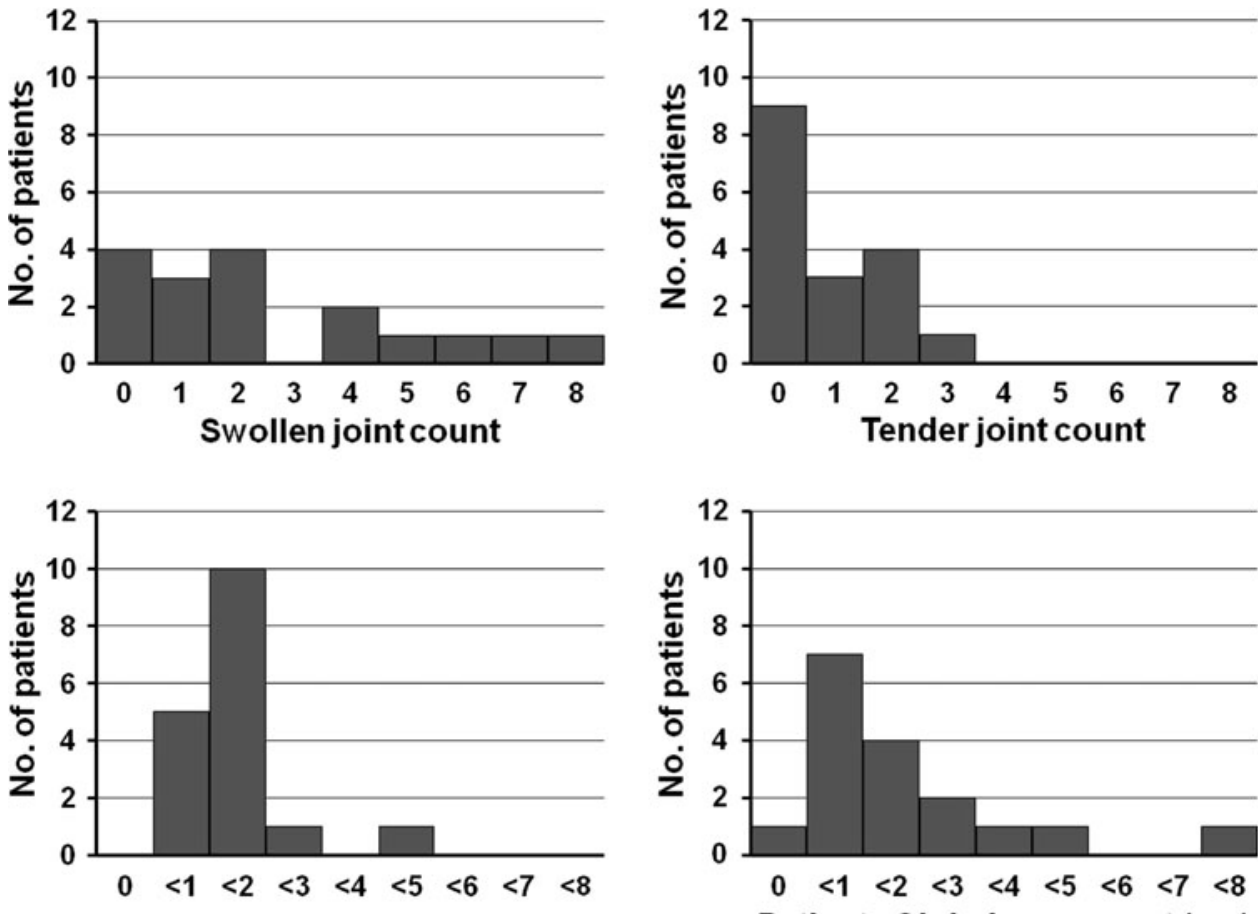

Physician Global assesment (cm)

Patients Global assesment (cm) was quite different from that based on the CDAI and SDAI, respectively. At week 24, DAS28-ESR remission was attained in $47.2 \%$ of the patients, even though the CDAI and SDAI remission rates were only 15.1 and $17.0 \%$, respectively (Table 2). To explore the reasons for the difference in these rates, we examined the characteristics of the patients who were in remission according to the DAS28 ("remitters") but not according to the CDAI or SDAI. Among the 17 patients who were in DAS28 remission but not CDAI remission, six patients (35.3\%) had a SJC $\geq 4$ and five patients $(31.5 \%)$ had a high patient global score $(>3 \mathrm{~cm}$; Fig. 4$)$. In contrast,
CDAI remitters generally had low scores on all measurements, and joint swelling and tenderness improved in most of these patients (Table 2). The same results were obtained when the distribution of each component of SDAI was analysed in the patients who attained DAS28 remission but not SDAI remission (Fig. 5; Table 2).

In the control (TCZ placebo plus MTX $8 \mathrm{mg} /$ week) group, only one patient (1.6\%) achieved even DAS28-ESR remission; this patient also achieved CDAI and SDAI remission. The number was too small and, therefore, a similar analysis was not available. 
Fig. 5 Histograms of SDAI remission variables in the 16 patients who attained remission according to the DAS28 but not according to the SDAI
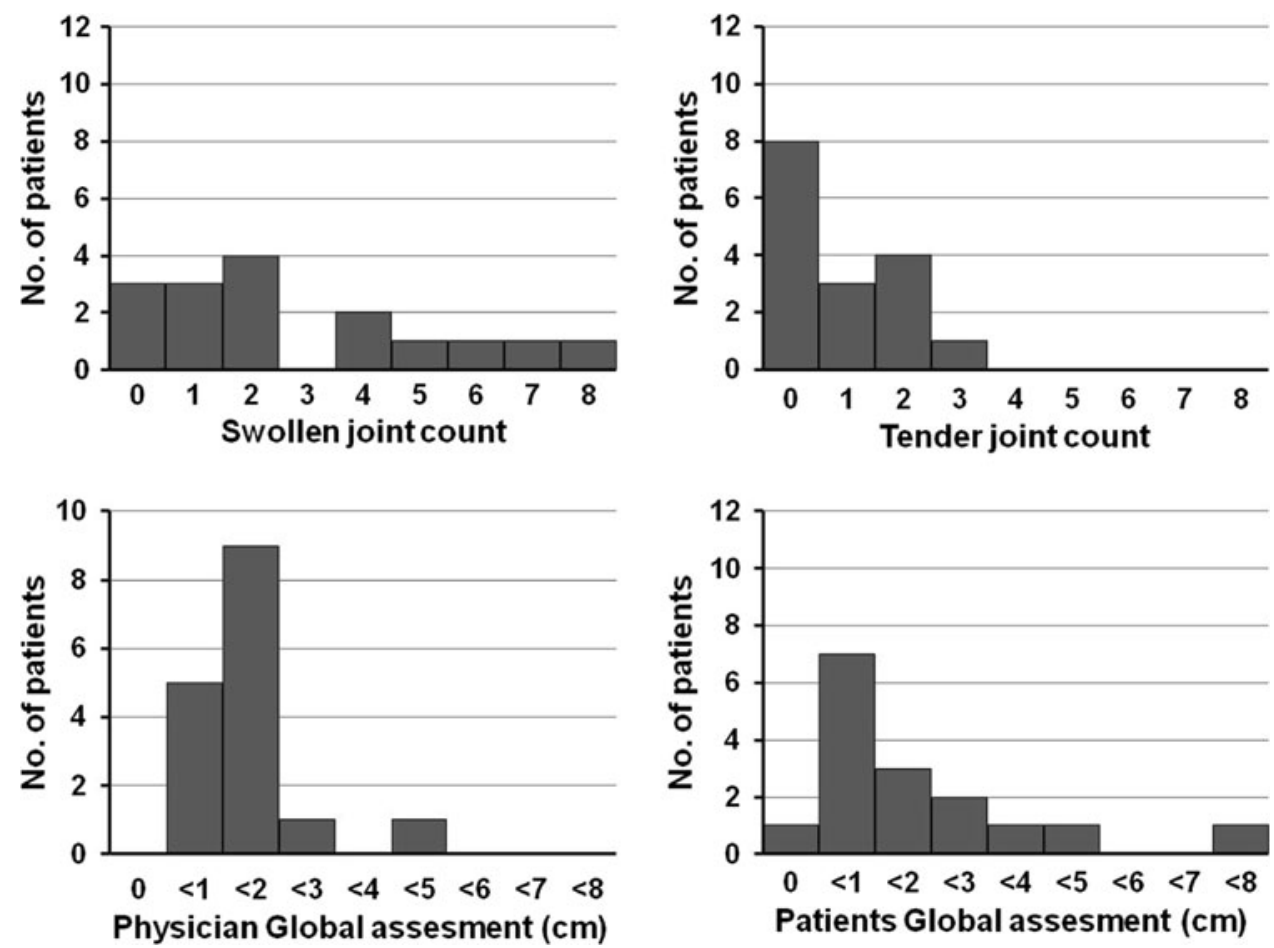

Correlation between ACR response category and changes in DAS28, CDAI and SDAI

Among the TCZ-treated patients who completed the 24week study, the ACR20, ACR50 and ACR70 response rates at week 24 were 83.0 (44/53), 54.7 (29/53) and 32.1\% (17/53), respectively. The CDAI and SDAI remitters (8 and 10 patients, respectively) all achieved ACR70. However, among the 25 DAS28-ESR remitters, 17 (68\%) achieved ACR70, three achieved ACR50 and five achieved only ACR20. No patient achieved DAS28-ESR remission without achieving at least ACR20. Among the DAS28ESR remitters who achieved ACR70, the maximum DAS28-ESR score was 2.43 .

\section{Discussion}

To compare the validity of methods of evaluating the antirheumatic effects of TCZ, an IL-6 receptor inhibitor that significantly reduces the level of inflammatory markers such as ESR and CRP, we investigated the correlations between DAS28-ESR and other methods for evaluating RA disease activity, namely, CDAI, SDAI and the ACR core set response category.

We found that the Spearman coefficient of correlation between DAS28-ESR and CDAI (whose formula does not include any inflammatory markers) at week 24 was $>0.8$ and that this correlation coefficient was comparable to those determined at baseline and weeks 4, 8, and 12, respectively. There was no decrease in the correlation coefficient related to the rapid improvement of ESR after the introduction of TCZ treatment. The same results were obtained when we compared the Spearman coefficient of correlation between DAS28-ESR and SDAI (whose formula includes the CRP level). The coefficients of correlation between DAS28-ESR and SDAI were also always $>0.8$. These results suggest that the DAS28-ESR score is not markedly lowered by the sudden decrease in ESR that occurs during the first 4 weeks of treatment with TCZ.

Shaver et al. [24] examined the correlation coefficient between DAS28 and CDAI among RA patients in the setting of a community rheumatology practice. Although all of the patients enrolled in their study did not receive TCZ treatment because TCZ had not yet been launched in the USA during the study period, the correlation coefficient obtained between these two indices was quite similar to our result. These researchers also reported that there was a large difference between the remission rate based on the DAS28 and that based on the CDAI and that only about $30 \%$ of DAS remitters could attain CDAI remission. The CDAI remitter and DAS remitter rates in our study were comparable with the respective rates in their study. These results suggest that a difference between DAS remission and CDAI remission is not specific to TCZ treatment, i.e., this difference was accrued due to the distinction in the definition of remission criterion in the DAS28-ESR and CDAI. These results also suggest that DAS28-ESR is as 
useful as CDAI in terms of evaluating the efficacy of TCZ and other anti-rheumatic drugs that have strong inhibiting effects on inflammatory markers.

Figure 4 provides insight into the factors responsible for the difference in remission rates according to the CDAI and DAS28 scales. Many of our patients who satisfied the DAS28 remission criteria but not the CDAI remission criteria had a high SJC and high PGA score. By nature of the definition of CDAI, CDAI remission patients generally had low scores on all of the incorporated measures. The same results were obtained when the difference in the remission rates according to the SDAI and DAS28 scales was analyzed even though the formula for SDAI includes the CRP level as a component for assessing remission (Fig. 5).

A difference between DAS28-ESR and CDAI or SDAI remission among ACR response rates was also observed. All CDAI and SDAI remitters achieved ACR70, but among the 25 DAS28-ESR remitters, 17 (61\%) achieved ACR70 while the others achieved no more than ACR50 or ACR20. Since all SDAI remitters achieved ACR70 even though the formula for SDAI includes an inflammatory marker (CRP), we concluded that the difference between the numbers of DAS28-ESR remitters and ACR70 responders was not caused by the inclusion of ESR in DAS28-ESR, but by the remission criterion used in the DAS28-ESR method. Sokka et al. [25] compared the performance of different definitions of remission, including DAS28, CDAI and ACR criteria. They concluded that the use of different definitions of RA remission leads to different results with regard to remission rate and that the rates of remission defined as "DAS28ESR $<2.6$ " are higher than those defined by other means. Mäkinen et al. [26] suggested that a stricter criterion (DAS28-ESR $<2.32$ ) is appropriate for defining DAS28ESR remission. Using the stricter criterion suggested by Mäkinen et al., all patients with DAS28-ESR $<2.32$ in the SATORI study achieved ACR70.

In conclusion, our results confirm that DAS28-ESR has a validity comparable to that of other methods in terms of evaluating the RA treatment efficacy of TCZ, even though this drug strongly inhibits inflammatory markers.

Acknowledgments The authors wish to thank the members of the MRA clinical study group for RA for treatment of the patients. This work was financially supported by Chugai Pharmaceutical Co., Ltd., Tokyo, Japan.

Conflict of interest statement NN, as a medical advisor, received a consulting fee and royalty for a systemic onset juvenile idiopathic arthritis patent from Chugai Pharmaceutical Co., Ltd., the product company of TCZ. He also works on the scientific advisory board of Hoffmann-La Roche who developed TCZ in collaboration with Chugai Pharmaceutical Co., Ltd. NT is an employee of Chugai Pharmaceutical Co., Ltd.

\section{References}

1. Mihara M, Kasutani K, Okazaki M, Nakamura A, Kawai S, Sugimoto M, et al. Tocilizumab inhibits signal transduction mediated by both mIL-6R and sIL-6R, but not by the receptors of other members of IL-6 cytokine family. Int Immunopharmacol. 2005;5:1731-40.

2. Nishimoto N, Yoshizaki K, Miyasaka N, Yamamoto K, Kawai S, Takeuchi $\mathrm{T}$, et al. Treatment of rheumatoid arthritis with humanized anti-interleukin- 6 receptor antibody: a multicenter, double-blind, placebo-controlled trial. Arthritis Rheum. 2004;50: 1761-9.

3. Nishimoto N, Hashimoto J, Miyasaka N, Yamamoto K, Kawai S, Takeuchi T, et al. Study of active controlled monotherapy used for rheumatoid arthritis, an IL-6 inhibitor (SAMURAI): evidence of clinical and radiographic benefit from an $\mathrm{x}$ ray reader-blinded randomised controlled trial of tocilizumab. Ann Rheum Dis. 2007;66:1162-7.

4. Nishimoto N, Miyasaka N, Yamamoto K, Kawai S, Takeuchi T, Azuma J, et al. Study of active controlled tocilizumab monotherapy for rheumatoid arthritis patients with an inadequate response to methotrexate (SATORI): significant reduction in disease activity and serum vascular endothelial growth factor by IL-6 receptor inhibition therapy. Mod Rheumatol. 2009;19:12-9.

5. Nishimoto N, Miyasaka N, Yamamoto K, Kawai S, Takeuchi T, Azuma J. Long-term safety and efficacy of tocilizumab, an antiIL-6 receptor monoclonal antibody, in monotherapy, in patients with rheumatoid arthritis (the STREAM study): evidence of safety and efficacy in a 5-year extension study. Ann Rheum Dis. 2009;68:1580-4.

6. Yokota S, Miyamae T, Imagawa T, Iwata N, Katakura S, Mori M, et al. Therapeutic efficacy of humanized recombinant anti-interleukin-6 receptor antibody in children with systemic-onset juvenile idiopathic arthritis. Arthritis Rheum. 2005;52:818-25.

7. Yokota S, Imagawa T, Mori M, Miyamae T, Aihara Y, Takei S, et al. Efficacy and safety of tocilizumab in patients with systemiconset juvenile idiopathic arthritis: a randomised, double-blind, placebo-controlled, withdrawal phase III trial. Lancet. 2008;371: 998-1006.

8. Nishimoto N, Kanakura Y, Aozasa K, Johkoh T, Nakamura M, Nakano S, et al. Humanized anti-interleukin-6 receptor antibody treatment of multicentric Castleman disease. Blood. 2005;106: 2627-32.

9. Mima T, Nishimoto N. Clinical value of blocking IL-6 receptor. Curr Opin Rheumatol. 2009;21:224-30.

10. Smolen JS, Beaulieu A, Rubbert-Roth A, Ramos-Remus C, Rovensky J, Alecock E, OPTION investigators, et al. Effect of interleukin-6 receptor inhibition with tocilizumab in patients with rheumatoid arthritis (OPTION study): a double-blind, placebocontrolled, randomised trial. Lancet. 2008;371:987-97.

11. Genovese MC, McKay JD, Nasonov EL, Mysler EF, da Silva NA, Alecock E, et al. Interleukin-6 receptor inhibition with tocilizumab reduces disease activity in rheumatoid arthritis with inadequate response to disease-modifying antirheumatic drugs: the tocilizumab in combination with traditional disease-modifying antirheumatic drug therapy study. Arthritis Rheum. 2008; 58:2968-80.

12. Emery P, Keystone E, Tony HP, Cantagrel A, van Vollenhoven $\mathrm{R}$, Sanchez A, et al. IL-6 receptor inhibition with tocilizumab improves treatment outcomes in patients with rheumatoid arthritis refractory to anti-tumour necrosis factor biologicals: results from a 24-week multicentre randomised placebo-controlled trial. Ann Rheum Dis. 2008;67:1516-23.

13. Jones G, Sebba A, Gu J, Lowenstein MB, Calvo A, et al. Comparison of tocilizumab monotherapy versus methotrexate 
monotherapy in patients with moderate to severe rheumatoid arthritis: the AMBITION study. Ann Rheum Dis. 2010;69:88-96.

14. Nishimoto N, Yoshizaki K, Maeda K, Kuritani T, Deguchi H, et al. Toxicity, pharmacokinetics, and dose-finding study of repetitive treatment with the humanized anti-interleukin 6 receptor antibody MRA in rheumatoid arthritis. Phase I/II clinical study. J Rheumatol. 2003;30:1426-35.

15. Prevoo ML, van 't Hof MA, Kuper HH, van Leeuwen MA, van de Putte LB, van Riel PL. Modified disease activity scores that include twenty-eight-joint counts. Development and validation in a prospective longitudinal study of patients with rheumatoid arthritis. Arthritis Rheum. 1995;38:44-8.

16. Aletaha D, Nell VP, Stamm T, Uffmann M, Pflugbeil S, Machold $\mathrm{K}$, et al. Acute phase reactants add little to composite disease activity indices for rheumatoid arthritis: validation of a clinical activity score. Arthritis Res Ther. 2005;7:R796-806.

17. Smolen JS, Breedveld FC, Schiff MH, Kalden JR, Emery P, Eberl $\mathrm{G}$, et al. A simplified disease activity index for rheumatoid arthritis for use in clinical practice. Rheumatology (Oxford). 2003;42:244-57.

18. Felson DT, Anderson JJ, Boers M, Bombardier C, Furst D, Goldsmith C, et al. American College of Rheumatology. Preliminary definition of improvement in rheumatoid arthritis. Arthritis Rheum. 1995;38:727-35.

19. Hochberg MC, Chang RW, Dwosh I, Lindsey S, Pincus T, Wolfe F. The American College of Rheumatology 1991 revised criteria for the classification of global functional status in rheumatoid arthritis. Arthritis Rheum. 1992;35:498-502.

20. van Gestel AM, Anderson JJ, van Riel PL, Boers M, Haagsma CJ, Rich B, et al. ACR and EULAR improvement criteria have comparable validity in rheumatoid arthritis trials. American College of Rheumatology European League of Associations for Rheumatology. J Rheumatol. 1999;26:705-11.

21. Fransen J, Creemers MC, Van Riel PL. Remission in rheumatoid arthritis: agreement of the disease activity score (DAS28) with the ARA preliminary remission criteria. Rheumatology (Oxford). 2004;43:1252-5.

22. Aletaha D, Funovits J, Keystone EC, Smolen JS. Disease activity early in the course of treatment predicts response to therapy after one year in rheumatoid arthritis patients. Arthritis Rheum. 2007;56:3226-35.

23. Aletaha D, Smolen JS. The simplified disease activity index (SDAI) and clinical disease activity index (CDAI) to monitor patients in standard clinical care. Best Pract Res Clin Rheumatol. 2007;21:663-75.

24. Shaver TS, Anderson JD, Weidensaul DN, Shahouri SS, Busch $\mathrm{RE}$, et al. The problem of rheumatoid arthritis disease activity and remission in clinical practice. J Rheumatol. 2008;35:1015-22.

25. Sokka T, Hetland ML, Mäkinen H, Kautiainen H, Hørslev-Petersen K, Luukkainen RK, Questionnaires in Standard Monitoring of Patients With Rheumatoid Arthritis Group, et al. Remission and rheumatoid arthritis: data on patients receiving usual care in twenty-four countries. Arthritis Rheum. 2008;58:2642-51.

26. Mäkinen H, Kautiainen H, Hannonen P, Sokka T. Is DAS28 an appropriate tool to assess remission in rheumatoid arthritis? Ann Rheum Dis. 2005;64:1410-3. 